\title{
New discrimination tools for harvest year and varieties of white wines based on hydrophilic interaction liquid chromatography with amperometric detection
}

Clara Pérez-Ràfols, Xavier Subirats, Núria Serrano*, José Manuel Díaz-Cruz

Department of Chemical Engineering and Analytical Chemistry, Universitat de Barcelona, Martí i Franquès 1-11, E-08028 Barcelona (Spain)

Phone: +34934033706, fax: +34934021233, e-mail: nuria.serrano@ub.edu

* to whom correspondence should be addressed

\section{ABSTRACT}

A simple HPLC-EC method based on hydrophilic interaction liquid chromatography with amperometric detection through gold screen-printed electrodes has been developed and applied for the first time to the determination of aminothiols in white wines. Moreover, the coupling of the method with partial least squares discriminant analysis (PLS-DA) using the analysed aminothiols as biomarkers provides wine discrimination in terms of harvest year. White wine samples were directly injected and chromatographic areas, together with $\mathrm{pH}$ and redox potential values, allowed a successful discrimination of wines from different harvest years with a global classification rate of $97.8 \%$. The developed HPLC-EC method also generated characteristic fingerprints that were combined with PLS-DA to classify wines according to three wine varieties, with a global classification rate of $95.3 \%$.

Keywords: HILIC; electrochemical detection; gold screen-printed electrode; aminothiols; wine analysis; partial least squares discriminant analysis 


\section{INTRODUCTION}

In recent years food quality and food authentication have become one of the most important aims for food industry and society since consumers give more value to the quality and origin of food products. Thus, labels like Protected Designation of Origin (PDO), local products and ecological products have gained more and more importance and status.

In this sense, one of the products in which these quality labels have been historically most valorised is wine. Regarding wine quality, both quality producers and final consumers usually pay special attention to sensory aspects that are usually related to wine variety, aging processes, style and aroma. In this sense, small soluble aminothiols such as glutathione, cysteine and related compounds have been reported as important biomarkers involved in the protection of white wines from both browning and losses of flavour and aroma $[1-4]$.

Considering the increasing importance of determining the occurrence of aminothiols in wine, numerous analytical methods have been developed for this purpose. The coexistence of different aminothiols in wine requires the use of separation techniques like chromatography or capillary electrophoresis. Particularly liquid chromatography coupled with UV-detection [5-9], fluorescence detection [10-12] or mass spectrometry [13-18] has been among the most exploited methods. However, UV and fluorescence detection methods require derivatisation treatments into UV absorbing or fluorescent species since these compounds present low UV-vis absorbance $[6,19,20]$ whereas the detection by means of mass spectrometry is quite expensive. A more affordable alternative detection method that can avoid tedious derivatisation procedures is electrochemical detection, which takes advantage of the electroactive character of 
aminothiols, being the oxidation of thiol groups to disulfide the main electrochemical reaction involved [21-28].

In electrochemical detection the selection of the amperometric sensor plays a key role. On the one hand, it has been reported that noble metals, like gold and silver, can be very convenient for the determination of aminothiols due to their affinity for thiol groups [27,29-31]. On the other hand, the use of screen-printed electrodes (SPE) has been postulated as a very convenient alternative to conventional solid electrodes due to their intrinsic characteristics, such as low-cost character and three-electrode configuration printed on the same strip [32]. Moreover, the disposable character of SPEs allows the possibility to work with a new electrode every day and, therefore avoids tedious polishing procedures. Thus, the coupling of liquid chromatography to electrochemical detection with disposable SPEs with a gold working electrode can be an interesting possibility for the determination of aminothiols.

Given the importance of these aminothiols in white wines, Sarakby et al [27] already developed a HPLC-EC method using a solid silver electrode for their determination. Taking advantage of this, the present study pretends to go one step further and not only determine aminothiols in wine, but also use them as biomarkers for wine aging processes. With this aim and taking into account the large amount of samples that should be considered in real scenarios, a HPLC-EC method based on isocratic elution of a simple mobile phase (aqueous trifluoroacetic acid and acetonitrile) with screen-printed electrodes as amperometric sensors was developed. Moreover, this method introduces, for the first time, hydrophilic interaction liquid chromatography (HILIC) to electrochemical detection, allowing wine analysis without any sample treatment. This HPLC-EC method was combined with chemometric strategies for the development of new discrimination tools not only for harvest year but also for white wine varieties. 


\section{EXPERIMENTAL}

\subsection{Chemicals and reagents}

Methanol, acetonitrile (ACN) (HPLC gradient grade, Fisher Scientific, Hampton, NH, USA), trifluoroacetic acid (TFA), ammonium acetate (Sigma-Aldrich, St. Louis, MO, USA) and mili-Q water (Millipore, Milford, MA, USA) were used for the preparation of mobile phases.

L-Cysteine (Cys, 99\%), DL-homocysteine (HCys, 95\%) and $N$-acetylcysteine (NAC, $\geq 99 \%$ ) were purchased from Sigma-Aldrich (St. Louis, MO, USA), whereas methionine (Met, 99\%) and reduced glutathione (GSH, 98\%) were supplied by Merck (Darmstadt, Germany). L (+)-ascorbic acid (AA, $\geq 99 \%$ ) was obtained from Riedel-de Haën (Seelze, Germany). Chemical structures of these compounds are shown in Scheme 1. 10 mmol L ${ }^{-1}$ standard stock solutions were prepared in Milli-Q water (Millipore, Milford, MA, USA) and stored at $4{ }^{\circ} \mathrm{C}$. Diluted standard solutions were prepared daily in $\mathrm{H}_{2} \mathrm{O}: \mathrm{ACN}(1: 1)$.

Sulphuric acid (Merck, Darmstadt, Germany) was used for electrode activation.

\subsection{Chromatographic system}

Chromatographic analyses were performed on an Agilent 1200 Series HPLC instrument equipped with a G1322A degasser, a G1311A quaternary pump, a G1329A autosampler, and a personal computer to control the HPLC system with the Agilent Chemstation software, all from Agilent Technologies (Palo Alto, CA, USA). An isocratic chromatographic separation at room temperature was achieved by: i) reversedphase mode with a $5 \mu \mathrm{m}, 25 \mathrm{~cm} \times 4.6 \mathrm{~mm}$ Ascentis C18 column supplied by Supelco (Bellefonte, PA, USA) fitted with an Agilent Eclipse XDB-C8 guard column (4.6 mm $\times$ 
$12.5 \mathrm{~mm}, 5 \mu \mathrm{m})$, using a mobile phase consisting of $0.05 \%(\mathrm{v} / \mathrm{v})$ TFA: ACN (98:2); and ii) HILIC mode by using a SeQuant ZIC-pHILIC $5 \mu \mathrm{m}$ polymer $150 \times 4.6 \mathrm{~mm}$ column (Merck, Darmstadt, Germany) with $0.05 \%$ (v/v) TFA: ACN (19:81) as a mobile phase. The flow rate was $1 \mathrm{~mL} \mathrm{~min}^{-1}$ and the injection volume was $5 \mu \mathrm{L}$.

The electrochemical detector was a HPLC electrochemical cell for SPEs (DRPHPLCELL) provided by Dropsens (Oviedo, Spain). The used SPEs were screen-printed gold electrodes (SPAuE) supplied by Dropsens (ref. 220AT), which comprise a threeelectrode configuration with a gold counter electrode, a silver pseudo-reference electrode, and a gold disk of $4 \mathrm{~mm}$ diameter as a working electrode. SPAuE was attached to a $\mu$ Autolab Type III (Ecochemie, The Netherlands) by means of a flexible cable (Dropsens, ref. CAC), which is connected to the personal computer with GPES version 4.9 software (Ecochemie). A new SPAuE device was used for every daily working session, and a conditioning potential of $-0.6 \mathrm{~V}$ was applied during $60 \mathrm{~s}$ before each measurement in order to prevent the adsorption of the thiol species on the gold surface [29], avoiding the loss of repeatability caused by the expected decrease of the electroactive surface area of the SPAuE.

Chromatographic peak areas were quantified using GPES version 4.9 software in the case of Met, HCys, Cys and GSH whereas NAC area was quantified by chemometric deconvolution using a home-made Matlab program based on the fitting of overlapping signals to the exponentially modified gaussian (EMG) function [33].

\subsection{Samples}

A total of 19 white wine samples, purchased in local supermarkets in November 2018, were considered for this study. This set of samples included white wines from three different grape varieties (6 Albariño, 6 Chardonnay, 6 Verdejo and 1 
Chardonnay/Albariño) and from vintages corresponding to two different years ( 8 from 2016 and 11 from 2017). For each wine sample, 2-3 replicates were analysed by HPLCEC without any sample pre-treatment and Oxidation Reduction Potential (ORP) and pH were measured.

ORP and $\mathrm{pH}$ measurements were carried out in a Crison micro pH $2000 \mathrm{pH}$-meter (Crison Instruments, Alella, Spain) using platinum and $\mathrm{pH}$ electrodes, respectively. Since ORP measurements are affected by oxygen exposure, some precautions in sample management were taken. Particularly, wine samples were opened and immediately fractionated into portions that were stored in sealed bottles at $4{ }^{\circ} \mathrm{C}$. ORP measurements were carried out in non-exposed portions in the shortest possible time and potential values are referred to $\mathrm{Ag} / \mathrm{AgCl}\left(3 \mathrm{~mol} \mathrm{~L}^{-1}\right)$.

\subsection{Data processing}

Data processing was based on partial least squares - discriminant analysis (PLS-DA) models. Three different data matrices were considered: (i) NAC, Met, HCys, Cys and GSH chromatographic peak areas; (ii) the previous peak areas together with $\mathrm{pH}$ and ORP values for models concerning vintages from different years; and (iii) chromatographic profiles from 0 to $10 \mathrm{~min}$ for models concerning wine varieties.

For the first two models, any possible variability caused by the use of different SPAuEs on different days was corrected. In each working session, a $10 \mu \mathrm{mol} \mathrm{L} \mathrm{L}^{-1}$ standard mixture was injected and the peak areas of the studied compounds were measured. Next, for each compound of the standard mixture the mean area for all sessions was calculated and then used to correct peak areas for all the samples injected in the same working session. In order to build these two models, the data were autoscaled and 2 latent variables (LV) were selected according to cross-validation. In the last model more 
complex pre-processing steps were performed through home-made programs implemented in Matlab, including baseline correction, compression by the discrete wavelet transform (DWT) method using Daubechies 4 wavelet function and a fourth decomposition level and autoscale. In this model 4 LVs were selected.

In all cases, data pre-processment, variable selection and construction of PLS-DA models were performed using Matlab® [34] with PLS-toolbox [35].

\section{RESULTS AND DISCUSSION}

\subsection{HPLC-EC optimization}

A standard mixture consisting of Cys, HCys, GSH, Met, AA and NAC at $25 \mu \mathrm{mol} \mathrm{L}^{-1}$ was analyzed by HPLC-EC. According to previous literature regarding aminothiol determination [21], their chromatographic separation was firstly evaluated by reversedphase liquid chromatography employing an Ascentis C18 column with an isocratic mobile phase composed of $0.05 \%(\mathrm{v} / \mathrm{v})$ TFA:methanol (98:2). A SPAuE applying a working electrode potential of $0.9 \mathrm{~V}$ was used as amperometric detector. However, the separation of the considered compounds was not fully achieved since AA and HCys coeluted. In order to improve this separation, considering that the mobile phase was mainly aqueous and increasing the content of organic modifier would only lead to shorter retention times without the desired resolution between the coeluting analytes, acetonitrile (ACN) was used as organic modifier instead of methanol. Under these conditions, $0.05 \%(\mathrm{v} / \mathrm{v})$ TFA:ACN (98:2), the separation of the six considered compounds was completely achieved.

Once the desired chromatographic separation was reached, the optimal working electrode conditions to perform the amperometric measurements of the considered 
compounds using the SPAuE were investigated. First, the SPAuE was immersed in 0.05 mol L-1 $\mathrm{H}_{2} \mathrm{SO}_{4}$ solution and 10 cyclic voltammetry $(\mathrm{CV})$ cycles from $0 \mathrm{~V}$ to $-1.25 \mathrm{~V}$ at $100 \mathrm{mV} \mathrm{s}^{-1}$ were applied in order to activate de sensor. This activation step provided a higher response for all the considered compounds (figure not shown). To optimize the working potential, chromatograms at different fixed potential values from $0.80 \mathrm{~V}$ to $1.05 \mathrm{~V}$ were measured. The highest response was obtained at $1.00 \mathrm{~V}$ for Cys, HCys, GSH and NAC, and $1.05 \mathrm{~V}$ for Met and AA (data not shown). Therefore, the most suitable working potential for the simultaneous determination of the considered compound was $1.00 \mathrm{~V}$ vs. $\mathrm{Ag} / \mathrm{AgCl}$ pseudoreference electrode, since at higher potential values the response decreases significantly, in particular for GSH and HCys. A representative chromatogram acquired by the optimized method after injection of $5 \mu \mathrm{L}$ of the standard mixture solution is presented in Figure 1a (inset). The retention times (min) of the considered compounds were: 2.78 for Cys, 3.52 for AA, 4.13 for HCys, 5.90 for GSH, 7.07 for Met and 10.62 for NAC.

Once the optimal HPLC-EC conditions were established for the mixture of standards, 5 $\mu \mathrm{L}$ of a wine sample were injected in order to test the effect of the wine matrix on the chromatographic behaviour of the studied analytes. As it is shown in the chromatograms presented in Figure 1a, components of the wine matrix elute in a broad unresolved band between 2 and 10 min, making impossible an accurate identification and integration of the Cys, AA, HCys, GSH, and Met peaks. Since the mobile phase had only a $2 \%$ ACN, it was not possible to increase the retention of the compounds of interest and separate them from the interfering components of the wine matrix by reducing the content of organic modifier. Thus, considering the molecular size, polar features and the acid-base properties of the studied analytes, a HILIC retention mode was selected instead of reversed-phase for the chromatographic separation. The chosen column was a ZIC- 
pHILIC, with a stationary phase consisting of a polymeric support functionalysed with zwitterionic sulfobetaine. Contrary to reversed-phase, in HILIC water is the strongest eluent of the mobile phase, and polar/ionized compounds are more retained than neutral ones [36]. At acidic $\mathrm{pH}$ the zwitterionic analytes Cys, HCys, GSH, and Met are expected to be positively charged, at least partially. Different chromatographic conditions were tested for HILIC in order to successfully separate the 6 analytes, injecting again a mixture of standards and adjusting the content of $\mathrm{ACN}$ and the $\mathrm{pH}$ of the mobile phase. TFA and ammonium acetate were the buffering species selected for the optimization study (data not shown). The final conditions were additionally refined in order to find the best possible separation between the unidentified wine components and the studied analytes, and this was reasonably achieved with a $0.05 \%$ TFA/ACN (19:81) mobile phase. Selectivity in HILIC is completely different from that of reversed-phase (Figure 1b). Met, HCys, Cys, and GSH eluted at the end of the wine matrix tail, while NAC was the less retained wine component. Unfortunately, AA appeared in the largest unresolved peak around $4 \mathrm{~min}$, and consequently this compound was excluded from further study.

\subsection{Linearity, sensitivity, limit of detection (LOD), limit of quantification (LOQ), repeatability and reproducibility,}

Once the optimal HPLC-EC conditions were stated, validation of the HPLC-EC method was performed through the evaluation of linearity, sensitivity, limit of detection (LOD), limit of quantification (LOQ), repeatability and reproducibility. For this purpose, a calibration curve for each aminothiol at seven concentration levels ranging from 0.20

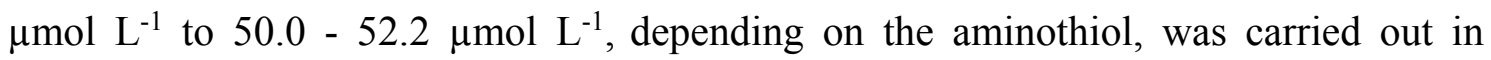
duplicate using two different SPAuEs. Calibration graphs were built using peak area vs. concentration of each considered aminothiol compound. The method performance is 
summarized in Table 1. Sensitivity was stated from the slopes of the calibration lines. A good linearity, assessed from the regression coefficients $\left(\mathrm{R}^{2}\right)$, was obtained for all the considered aminothiols. The minimum concentration of aminothiols that the HPLC-EC method can detect and quantify was assessed from the LOD and LOQ values, respectively. LOD and LOQ are computed as 3 and 10 times the standard deviation of the intercept over the slope of the calibration curves of the aminothiol compounds, respectively. The obtained LODs for the five aminothiols ranged from $0.52 \mu \mathrm{mol} \mathrm{L}^{-1}$ to $1.26 \mu \mathrm{mol} \mathrm{L} \mathrm{L}^{-1}$ (Table 1), whereas LOQ varied from $1.74 \mu \mathrm{mol} \mathrm{L}^{-1}$ to $4.19 \mu \mathrm{mol} \mathrm{L}^{-1}$ (Table 1). These results, compared to previous values reported in the literature [21,27], are similar or even slightly better depending on both the studied aminothiol and the considered linear range.

Repeatability (intra-day) was estimated from five repetitive measurements of a solution containing $10 \mu \mathrm{mol} \mathrm{L} \mathrm{L}^{-1}$ of each considered compound using the same SPAuE unit. Repeatability values, expressed as RSD \%, ranged from 1.98 to 2.85 depending on the considered aminothiol. Reproducibility (inter-day) was calculated from the slopes of two calibration curves obtained using two different SPAuE units, with RSD \% values ranging from 0.95 to 6.74 depending again on the considered aminothiol. Both repeatability and reproducibility obtained values are very good and significantly better than those reported in the literature for aminothiols determination [21,24].

The obtained results show that the proposed HPLC-EC method is very satisfactory in terms of linearity, sensitivity and precision for the determination of aminothiols. Regarding repeatability and reproducibility, the results obtained demonstrated that a renewed electrode surface is obtained after the application of the conditioning potential of $-0.6 \mathrm{~V}$ during $60 \mathrm{~s}$, which is enough to prevent thiol adsorption on the electrode surface throughout a daily working session. This, coupled to the disposable and low- 
cost character of SPEs, which makes possible the use of a new electrode every day, avoids more aggressive pretreatments such as polishing or cutting a thin slide of the surface between measurements. Moreover, the use of SPAuE as amperometric sensor provides the additional advantages attributed to the SPEs i.e. commercial availability, miniaturized size and its ease of use due to the characteristic three-electrode configuration that prevents the use of any external reference or counter electrode.

\subsection{Discrimination between vintages from different years}

Thiolic compounds are known to degrade over time during aging of white wines due to both oxidation by molecular oxygen and addition reactions with o-quinones generated during oxidation of polyphenols [37]. Therefore, the concentration of the studied aminothiols could be used to discriminate between vintages from different years.

In order to test this hypothesis, the proposed HPLC-EC method was applied to 8 and 11 white wines from the 2016 and 2017 vintages respectively and the peak areas of NAC, Met, HCys, Cys and GSH were used to build a PLS-DA model. For this model the samples were divided into calibration (5 wines from 2016 and 7 wines from 2017) and validation sets (3 wines from 2016 and 4 wines from 2017) and 2-3 replicates were included for each sample. The combined scores and loadings plot is presented in Figure 2a. As it can be seen, there is a clear tendency of separation between vintages from 2016 and 2017 given by lower amounts of aminothiols in wines from 2016, which is consistent with a decrease of these compounds during wine aging. This decrease is particularly important in the case of GSH, in which values range from 4.5 to $16.7 \mu \mathrm{mol}$ $\mathrm{L}^{-1}$ in wines from 2017 and from 2.84 to $9.30 \mu \mathrm{mol} \mathrm{L}^{-1}$ in wines from 2016 . However, some 2016 and 2017 samples are overlapped in the border area between classes, which results in a total of 6 replicates, mainly from 2017, being misclassified (Table 2). In 
terms of numerical performance, the global classification rate (total samples correctly assigned) was $87.0 \%$, and average sensitivity (samples of each class correctly assigned) and specificity (samples of other classes correctly rejected) were both $87.9 \%$. It should also be pointed out that the samples corresponding to the 2016 vintage are closer to each other. This indicates that, although aminothiols can be initially found in different proportions in white wine, the aging process tends to homogenize them.

In order to achieve a better discrimination between vintages from different years, two more parameters, $\mathrm{pH}$ and ORP, were considered. For all the white wines considered $\mathrm{pH}$ and ORP ranged from 2.69 to 3.13 and from 148 to $224 \mathrm{mV}$, respectively. These new data were combined with the corresponding aminothiols peak areas to build a new PLSDA model. Figure $2 \mathrm{~b}$ and Table 2 show, respectively, the combined scores and loadings plot and the confusion matrix resulting from this model. It can be observed that a much better discrimination is achieved, with only one sample from 2017 being misclassified. This results in a global classification rate of $97.8 \%$ with both an average sensitivity and specificity of $98.1 \%$. As it can be observed from the loadings, $\mathrm{pH}$ plays a much more important role in the discrimination between classes than ORP. In this sense, wine samples from grapes harvested in 2016 tend to be more acidic than those collected in 2017. A possible explanation for this fact could be that a lower $\mathrm{pH}$ slows the loss of wine sensorial qualities because it decreases the rate of autoxidation of phenolic compounds [38]. Therefore, less acidic wines would not be considered good candidates to undergo an aging process.

Then, the combination of the HPLC-EC method with potentiometric measurements together with chemometric strategies allows the successful discrimination of young and aged white wines. It should be pointed out that both HPLC-EC and potentiometric measurements involved in this model have the advantage of not requiring any sample 
treatment. In particular, it is worth noting that in this HPLC-EC method the wine sample can be directly injected without neither derivatization, dilution nor extraction treatments.

\subsection{Classification of wines from different grape varieties}

In addition to the determination of the five aminothiols studied, the proposed HPLC-EC method also allowed the comparison of LC-profiles of different wines. In this sense, it was observed that different white wine varieties, namely Albariño, Verdejo and Chardonnay, showed significant differences in profiles, particularly in the region $0-10$ min (Figure 3). Therefore, the classification of white wine samples from these three varieties was attempted using this region of the obtained LC-profiles. For this purpose, wine samples were distributed between a calibration (4 Albariño, 4 Verdejo and 4 Chardonnay) and a validation set (2 Albariño, 2 Verdejo, 2 Chardonnay and 1 Albariño/Chardonnay) and a PLS-DA model was built. The resulting 3D scores plot is shown in Figure 4. In the scores plot in LV1, LV3 and LV4 (Figure 4a) Verdejo samples can be easily distinguished whereas the scores plot in LV2, LV3 and LV4 (Figure 4b) allows the discrimination between Albariño and Chardonnay samples. It should also be pointed out that the Albariño/Chardonnay sample is mainly associated to the rest of the Albariño samples and, in both representations, closer to Chardonnay samples than Verdejo ones.

The numerical performance of this PLS-DA model was also assessed in terms of classification rate, sensitivity and specificity. The resulting confusion matrix is presented in Table 3. As it was previously observed in the plots, most of the samples were correctly assigned, with only 2 replicates of an Albariño wine being misclassified. 
A global classification rate of $95.3 \%$ was achieved, with an average sensitivity and specificity of $95.2 \%$ and $97.6 \%$, respectively.

In summary, these results suggest that the proposed HPLC-EC method using SPAuE as amperometric sensor allows the successful classification of white wines according to their varieties. Although other methods like electronic tongues have also been reported for the classification of wines $[39,40]$, the presented method has the advantage of using only one commercial sensor in contrast to an array of sensors that require a crossresponse, which often implies the use of modified electrodes. Moreover, the linear character of chromatographic data allows the use of linear chemometric methods such as PLS-DA and avoids the use of more complex chemometric tools like artificial neural networks.

\section{CONCLUSIONS}

In this work a HPLC-EC method based on hydrophilic interaction liquid chromatography using SPAuE as amperometric sensor was for the first time developed and optimized for the determination of five aminothiols and applied to white wine analysis. It should be highlighted that the proposed method does not require any sample pre-treatment since the wine can be directly injected. This method was fully validated through sensitivity, linearity, LOD, LOQ, repeatability and reproducibility, with similar or even better performance than other previously reported methods based on reversedphase chromatography. Moreover, this new method takes advantage from the use of SPEs, which not only can be used for a large number of measurements without signs of deterioration but can also avoid tedious polishing procedures, which are mandatory in the case of solid electrodes. 
The measured area of these five aminothiols in combination with the use of chemometric tools such as PLS-DA showed a clear tendency of discrimination between vintages from two different years, namely 2016 and 2017, with a global classification rate of $87.0 \%$. This model was clearly improved with the addition of two new parameters, $\mathrm{pH}$ and ORP, that allowed the construction of a new PLS-DA model that increased the global classification rate up to $97.8 \%$. From the resulting loadings of this model it can be stated that GSH area and $\mathrm{pH}$ were the two parameters with the highest contribution to this classification.

Moreover, the fingerprinting study of the wine LC-profiles allowed the classification of white wines from three different grape varieties. For this classification, the measured chromatograms were compressed employing DWT and PLS-DA models, achieving a global classification rate of $95.3 \%$.

\section{Acknowledgments}

This work is supported by the Generalitat of Catalonia (Project 2017SGR311). The authors thank Merck KGaA (Darmstadt, Germany) and Dr. Patrik Appelblad for providing the SeQuant ZIC-pHILIC column. Clara Pérez-Ràfols acknowledges the Spanish Ministry of Education, Culture and Sports for a Ph.D grant (reference FPU15/02140) for its support. Xavier Subirats thanks the support of the Ministry of Science, Innovation and Universities of Spain (project CTQ2017-88179-P AEI/FEDER, UE).

\section{References}

[1] C. Coetzee, W.J. du Toit, A comprehensive review on Sauvignon blanc aroma 
with a focus on certain positive volatile thiols, Food Res. Int. 45 (2012) 287-298. doi:10.1016/j.foodres.2011.09.017.

[2] E.C. Kritzinger, F.F. Bauer, W.J. Du Toit, Role of glutathione in winemaking: A review, J. Agric. Food Chem. 61 (2013) 269-277. doi:10.1021/jf303665z.

[3] I.G. Roussis, D. Papadopoulou, M. Sakarellos-Daitsiotis, Protective effect of thiols on wine aroma volatiles, Open Food Sci. J. 3 (2009) 98-102. doi:10.2174/1874256400903010098.

[4] A. Peña-Gallego, P. Hernández-Orte, J. Cacho, V. Ferreira, S-Cysteinylated and S-glutathionylated thiol precursors in grapes. A review, Food Chem. 131 (2012) 1-13. doi:10.1016/j.foodchem.2011.07.079.

[5] E. Bald, G. Chwatko, R. Głowacki, K. Kuśmierek, Analysis of plasma thiols by high-performance liquid chromatography with ultraviolet detection, J. Chromatogr. A. 1032 (2004) 109-115. doi:10.1016/j.chroma.2003.11.030.

[6] E. Camera, M. Picardo, Analytical methods to investigate glutathione and related compounds in biological and pathological processes, J. Chromatogr. B. 781 (2002) 181-206. doi:10.1016/S1570-0232(02)00618-9.

[7] K. Amarnath, V. Amarnath, K. Amarnath, H.L. Valentine, W.M. Valentine, A specific HPLC-UV method for the determination of cysteine and related aminothiols in biological samples, Talanta. 60 (2003) 1229-1238. doi:10.1016/S0039-9140(03)00232-7.

[8] E. Bald, E. Kaniowska, G. Chwatko, R. Glowacki, Liquid chromatographic assessment of total and protein-bound homocysteine in human plasma, Talanta. 50 (2000) 1233-1243. doi:10.1016/S0039-9140(99)00235-0. 
[9] K. Kuśmierek, E. Bald, Reversed-phase liquid chromatography method for the determination of total plasma thiols after derivatization with 1-benzyl-2chloropyridinium bromide, Biomed. Chromatogr. 23 (2009) 770-775. doi:10.1002/bmc.1183.

[10] G. Cevasco, A.M. Piątek, C. Scapolla, S. Thea, An improved method for simultaneous analysis of aminothiols in human plasma by high-performance liquid chromatography with fluorescence detection, J. Chromatogr. A. 1217 (2010) 2158-2162. doi:10.1016/J.CHROMA.2010.02.012.

[11] R. Ferin, M.L. Pavão, J. Baptista, Methodology for a rapid and simultaneous determination of total cysteine, homocysteine, cysteinylglycine and glutathione in plasma by isocratic RP-HPLC, J. Chromatogr. B. 911 (2012) 15-20. doi:10.1016/J.JCHROMB.2012.10.022.

[12] S.-T. Chou, L.-E. Ko, C.-S. Yang, High performance liquid chromatography with fluorimetric detection for the determination of total homocysteine in human plasma: method and clinical applications, Anal. Chim. Acta. 429 (2001) 331336. doi:10.1016/S0003-2670(00)01296-4.

[13] J.H. Suh, R. Kim, B. Yavuz, D. Lee, A. Lal, B.N. Ames, et al., Clinical assay of four thiol amino acid redox couples by LC-MS/MS: Utility in thalassemia, J. Chromatogr. B. 877 (2009) 3418-3427. doi:10.1016/J.JCHROMB.2009.06.041.

[14] M. Rafii, R. Elango, G. Courtney-Martin, J.D. House, L. Fisher, P.B. Pencharz, High-throughput and simultaneous measurement of homocysteine and cysteine in human plasma and urine by liquid chromatography-electrospray tandem mass spectrometry, Anal. Biochem. 371 (2007) 71-81. doi:10.1016/J.AB.2007.07.026. 
[15] X. Guan, B. Hoffman, C. Dwivedi, D.P. Matthees, A simultaneous liquid chromatography/mass spectrometric assay of glutathione, cysteine, homocysteine and their disulfides in biological samples, J. Pharm. Biomed. Anal. 31 (2003) 251-261. doi:10.1016/S0731-7085(02)00594-0.

[16] M.H.A. El-Zohri, R. Cabala, H. Frank, Quantification of phytochelatins in plants by reversed-phase HPLC-ESI-MS-MS, Anal. Bioanal. Chem. 382 (2005) 18711876. doi:10.1007/s00216-005-3331-0.

[17] B. Klejdus, J. Zehnálek, V. Adam, J. Petřek, R. Kizek, J. Vacek, et al., Subpicomole high-performance liquid chromatographic/mass spectrometric determination of glutathione in the maize (Zea mays L.) kernels exposed to cadmium, Anal. Chim. Acta. 520 (2004) 117-124. doi:10.1016/J.ACA.2004.02.060.

[18] E. Camera, M. Rinaldi, S. Briganti, M. Picardo, S. Fanali, Simultaneous determination of reduced and oxidized glutathione in peripheral blood mononuclear cells by liquid chromatography-electrospray mass spectrometry, J. Chromatogr. B Biomed. Sci. Appl. 757 (2001) 69-78. doi:10.1016/S03784347(01)00081-0.

[19] M. Özyürek, S. Baki, N. Güngör, S.E. Çelik, K. Güçlü, R. Apak, Determination of biothiols by a novel on-line HPLC-DTNB assay with post-column detection, Anal. Chim. Acta. 750 (2012) 173-181. doi:10.1016/J.ACA.2012.03.056.

[20] T. Toyo'oka, Recent advances in separation and detection methods for thiol compounds in biological samples, J. Chromatogr. B. 877 (2009) 3318-3330. doi:10.1016/J.JCHROMB.2009.03.034. 
[21] S. Cavanillas, N. Serrano, J.M. Díaz-Cruz, C. Ariño, M. Esteban, Commercial screen-printed gold electrodes for the detection and quantification of aminothiols in human plasma by liquid chromatography with electrochemical detection, Electroanalysis. 26 (2014) 581-587. doi:10.1002/elan.201300593.

[22] D. Potesil, J. Petrlova, V. Adam, J. Vacek, B. Klejdus, J. Zehnalek, et al., Simultaneous femtomole determination of cysteine, reduced and oxidized glutathione, and phytochelatin in maize (Zea mays L.) kernels using highperformance liquid chromatography with electrochemical detection, J. Chromatogr. A. 1084 (2005) 134-144. doi:10.1016/J.CHROMA.2005.06.019.

[23] W.A. Kleinman, J.P. Richie, Determination of thiols and disulfides using highperformance liquid chromatography with electrochemical detection, J.

Chromatogr. B Biomed. Sci. Appl. 672 (1995) 73-80. doi:10.1016/03784347(94)00194-A.

[24] A. Khan, M.I. Khan, Z. Iqbal, Y. Shah, L. Ahmad, S. Nazir, et al., A new HPLC method for the simultaneous determination of ascorbic acid and aminothiols in human plasma and erythrocytes using electrochemical detection, Talanta. 84 (2011) 789-801. doi:10.1016/j.talanta.2011.02.019.

[25] À. Dago, O. González-García, C. Ariño, J.M. Díaz-Cruz, M. Esteban, Liquid chromatographic analysis of $\mathrm{Hg}(\mathrm{II})$ binding by thiol-rich peptides using both UV-vis and electrochemical detection, J. Chromatogr. A. 1216 (2009) 67526757. doi:10.1016/J.CHROMA.2009.07.071.

[26] S. Zhang, W. Sun, Y. Xian, W. Zhang, L. Jin, K. Yamamoto, et al., Multichannel amperometric detection system for liquid chromatography to assay the thiols in human whole blood using the platinum microelectrodes chemically modified by 
copper tetraaminophthalocyanine, Anal. Chim. Acta. 399 (1999) 213-221. doi:10.1016/S0003-2670(99)00467-5.

[27] A. Sarakbi, Z. Aydogmus, A. Dago, D. Mertens, J.Y. Dewert, J.M. Kauffmann, Determination of aminothiols by liquid chromatography with amperometric detection at a silver electrode: Application to white wines, Anal. Chim. Acta. 786 (2013) 22-28. doi:10.1016/j.aca.2013.04.070.

[28] P. Jakubec, V. Halouzka, J. Skopalova, J. Hrbac, Electrode modified with cobalt cyclohexylbutyrate for the determination of low molecular weight thiol group bearing compounds using catalytic stripping voltammetry, Electroanalysis. 22 (2010) 345-350. doi:10.1002/elan.200900309.

[29] E. Chekmeneva, J.M. Díaz-Cruz, C. Ariño, M. Esteban, Binding of $\mathrm{Hg}^{2+}$ by Cys, Cys-Gly and reduced glutathione: Study by differential pulse voltammetry on rotating Au-disk electrode, electrospray ionization mass-spectrometry and isothermal titration calorimetry, J. Electroanal. Chem. 644 (2010) 20-24. doi:10.1016/j.jelechem.2010.03.021.

[30] L. Agüí, C. Peña-Farfal, P. Yáñez-Sedeño, J.M. Pingarrón, Electrochemical determination of homocysteine at a gold nanoparticle-modified electrode, Talanta. 74 (2007) 412-420. doi:10.1016/J.TALANTA.2007.05.035.

[31] L.-H. Wang, W.-S. Huang, L.-H. Wang, W.-S. Huang, Electrochemical oxidation of cysteine at a film gold modified carbon fiber microelectrode. Its application in a flow-through voltammetric sensor, Sensors. 12 (2012) 3562-3577. doi:10.3390/s120303562.

[32] J. Barton, M.B.G. García, D.H. Santos, P. Fanjul-Bolado, A. Ribotti, M. McCaul, 
et al., Screen-printed electrodes for environmental monitoring of heavy metal ions: a review, Microchim. Acta. 183 (2016) 503-517. doi:10.1007/s00604-015$1651-0$

[33] S. Cavanillas, N. Serrano, J.M. Díaz-Cruz, C. Ariño, M. Esteban, Parametric signal fitting of highly asymmetric voltammograms by using the exponentially modified Gaussian (EMG) function, Chemom. Intell. Lab. Syst. 152 (2016) 8087. doi:10.1016/j.chemolab.2016.01.012.

[34] Matlab, version R2008b ed., Mathworks Inc.: Natick, MA, USA, (2008).

[35] PLS-toolbox version 7.8.2 (Eigenvector Research Inc., Wenatchee, USA), (n.d.).

[36] T. Alvarez-Segura, X. Subirats, M. Rosés, Retention-pH profiles of acids and bases in hydrophilic interaction liquid chromatography, Anal. Chim. Acta. 1050 (2019) 176-184. doi:10.1016/j.aca.2018.11.021.

[37] A. Sarakbi, J.M. Kauffmann, A new chemical criteria for white wine: The glutathione equivalent capacity, Food Chem. 153 (2014) 321-326. doi:10.1016/j.foodchem.2013.12.090.

[38] A.C. Silva Ferreira, P. Guedes de Pinho, P. Rodrigues, T. Hogg, Kinetics of oxidative degradation of white wines and how they are affected by selected technological parameters, J. Agric. Food Chem. 50 (2002) 5919-5924. doi:10.1021/nn3017052.

[39] J. Zeravik, A. Hlavacek, K. Lacina, P. Skládal, State of the art in the field of electronic and bioelectronic tongues - towards the analysis of wines, Electroanalysis. 21 (2009) 2509-2520. doi:10.1002/elan.200900285. 
[40] X. Cetó, J. Capdevila, A. Puig-Pujol, M. del Valle, Cava wine authentication employing a voltammetric electronic tongue, Electroanalysis. 26 (2014) 15041512. doi:10.1002/elan.201400057. 


\section{TABLES}

Table 1. Calibration parameters, repeatability and reproducibility of NAC, Met, HCys, Cys and GSH obtained using the proposed chromatographic method. The standard deviations are denoted by parenthesis.

\begin{tabular}{lccccc}
\hline & NAC & Met & HCys & Cys & GSH \\
\hline${\text { Sensitivity }\left(\mathrm{nC} \mu \mathrm{mol}^{-1} \mathrm{~L}\right)}^{63.4(0.6)}$ & $27.8(0.5)$ & $49.7(0.4)$ & $54.5(0.8)$ & $38.5(0.4)$ \\
$\mathrm{R}^{2}$ & 0.9990 & 0.9970 & 0.9995 & 0.9980 & 0.9991 \\
Linear range $^{\mathrm{a}}\left(\mu \mathrm{mol} \mathrm{L} \mathrm{L}^{-1}\right)$ & $2.03-52.2$ & $4.19-50.1$ & $1.74-50.6$ & $3.22-50.0$ & $2.81-50.6$ \\
$\mathrm{LOD}\left(\mu \mathrm{mol} \mathrm{L} \mathrm{L}^{-1}\right)$ & 0.61 & 1.26 & 0.52 & 0.97 & 0.84 \\
Repeatability $(\%)$ & 2.14 & 2.85 & 2.14 & 1.98 & 2.45 \\
Reproducibility $(\%)$ & 1.90 & 6.74 & 0.95 & 2.15 & 3.07 \\
\hline
\end{tabular}

${ }^{a}$ The lowest value of the linear range was considered from LOQ

Table 2. Confusion matrix resulting from the year classes assigned by the PLS-DA models for both calibration and validation sets.

\begin{tabular}{|c|c|c|c|c|}
\hline \multicolumn{5}{|l|}{ Aminothiol areas } \\
\hline & \multicolumn{2}{|c|}{ Calibration set } & \multicolumn{2}{|c|}{ Validation set } \\
\hline & Expected 2016 & Expected 2017 & Expected 2016 & Expected 2017 \\
\hline Predicted 2016 & 12 & 3 & 7 & 2 \\
\hline Predicted 2017 & 1 & 13 & 0 & 8 \\
\hline \multicolumn{5}{|c|}{ Aminothiol areas, pH and ORP } \\
\hline & \multicolumn{2}{|c|}{ Calibration set } & \multicolumn{2}{|c|}{ Validation set } \\
\hline & Expected 2016 & Expected 2017 & Expected 2016 & Expected 2017 \\
\hline Predicted 2016 & 13 & 1 & 7 & 0 \\
\hline Predicted 2017 & 0 & 15 & 0 & 10 \\
\hline
\end{tabular}

Table 3. Confusion matrix resulting from the varietal classes assigned by the PLS-DA models for both calibration and validation sets.

\begin{tabular}{ccccccc}
\hline & \multicolumn{3}{c}{ Calibration set } & \multicolumn{3}{c}{ Validation set } \\
\cline { 2 - 7 } & $\begin{array}{c}\text { Expected } \\
\text { Albariño }\end{array}$ & $\begin{array}{c}\text { Expected } \\
\text { Chardonnay }\end{array}$ & $\begin{array}{c}\text { Expected } \\
\text { Verdejo }\end{array}$ & $\begin{array}{c}\text { Expected } \\
\text { Albariño }\end{array}$ & $\begin{array}{c}\text { Expected } \\
\text { Chardonnay }\end{array}$ & $\begin{array}{c}\text { Expected } \\
\text { Verdejo }\end{array}$ \\
\hline $\begin{array}{c}\text { Predicted } \\
\text { Albariño }\end{array}$ & 9 & 0 & 0 & 3 & 0 & 0 \\
$\begin{array}{c}\text { Predicted } \\
\text { Chardonnay }\end{array}$ & 0 & 10 & 0 & 0 & 4 & 0 \\
$\begin{array}{c}\text { Predicted } \\
\text { Verdejo }\end{array}$ & 0 & 0 & 10 & 2 & 0 & 5 \\
\hline
\end{tabular}




\section{FIGURES}<smiles>NC(CS)C(=O)O</smiles>

Cysteine (Cys)<smiles>NC(CCS)C(=O)O</smiles>

Homocysteine (Hcys)<smiles>CSCCC(N)C(=O)O</smiles>

Methionine (Met)<smiles>CC(=O)NC(CS)C(=O)O</smiles>

$\mathrm{N}$-acetyl-cysteine (NAC)<smiles>O=C1OC(C(O)CO)C(O)=C1O</smiles>

Ascorbic acid (AA)<smiles>NC(CCC(=O)NC(CS)C(=O)NCC(=O)O)C(=O)O</smiles>

Glutathione (GSH)

Scheme 1. Chemical structures of the studied compounds. 

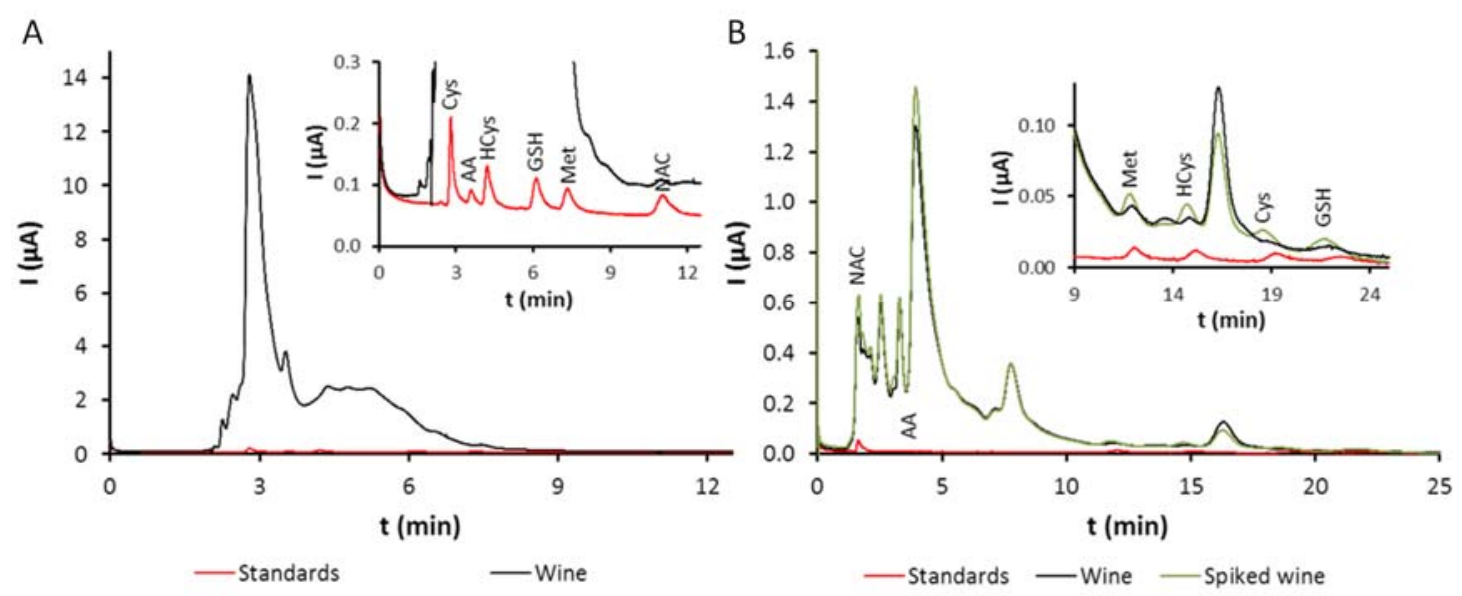

Figure 1. Representative HPLC-EC chromatograms of A) $25 \mu \mathrm{mol} \mathrm{L}{ }^{-1}$ thiol standard solution and white wine using an Ascentis C18 column with a mobile phase of $0.05 \%$ (v/v) TFA: ACN (98:2) and a flow rate of $1 \mathrm{~mL} \mathrm{~min}^{-1}$ and B) $10 \mu \mathrm{mol} \mathrm{L}^{-1}$ thiol standards, wine and wine spiked with $25 \mu \mathrm{mol} \mathrm{L}{ }^{-1}$ thiols using a SeQuant ZIC-pHILIC column with a mobile phase of $0.05 \%$ (v/v) TFA: ACN (19:81) and a flow rate of $1 \mathrm{~mL}$ $\min ^{-1}$.
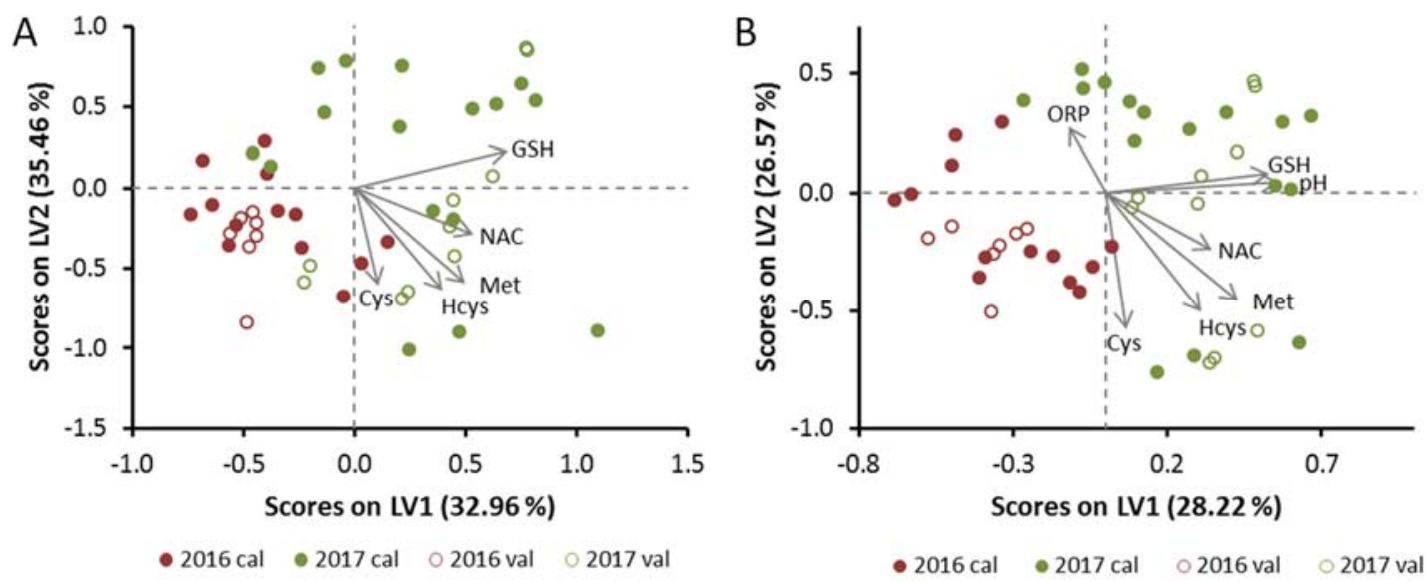

Figure 2. Combined scores and loadings plots obtained after PLS-DA models constructed from (A) NAC, Met, HCys, Cys and GSH chromatographic areas, and (B) the previous chromatographic areas together with $\mathrm{pH}$ and ORP. Calibration (cal) and validation (val) sets are represented by filled and empty symbols respectively. 


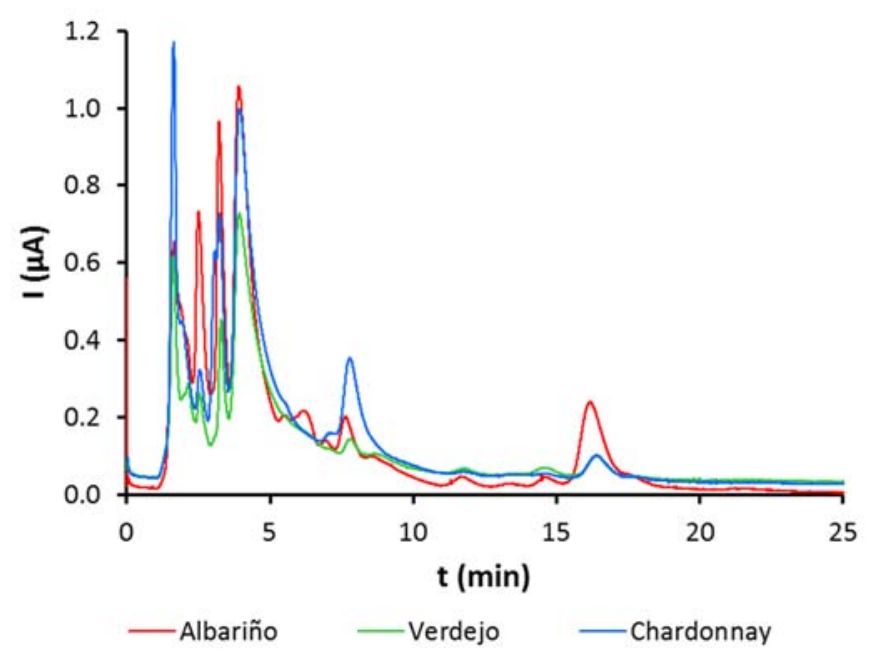

Figure 3. Representative HPLC-EC chromatograms of the three white wine grape varieties considered (Albariño, Verdejo and Chardonnay) using a SeQuant ZIC-pHILIC column with a mobile phase of $0.05 \%$ (v/v) TFA: ACN (19:81) and a flow rate of $1 \mathrm{~mL}$ $\min ^{-1}$.
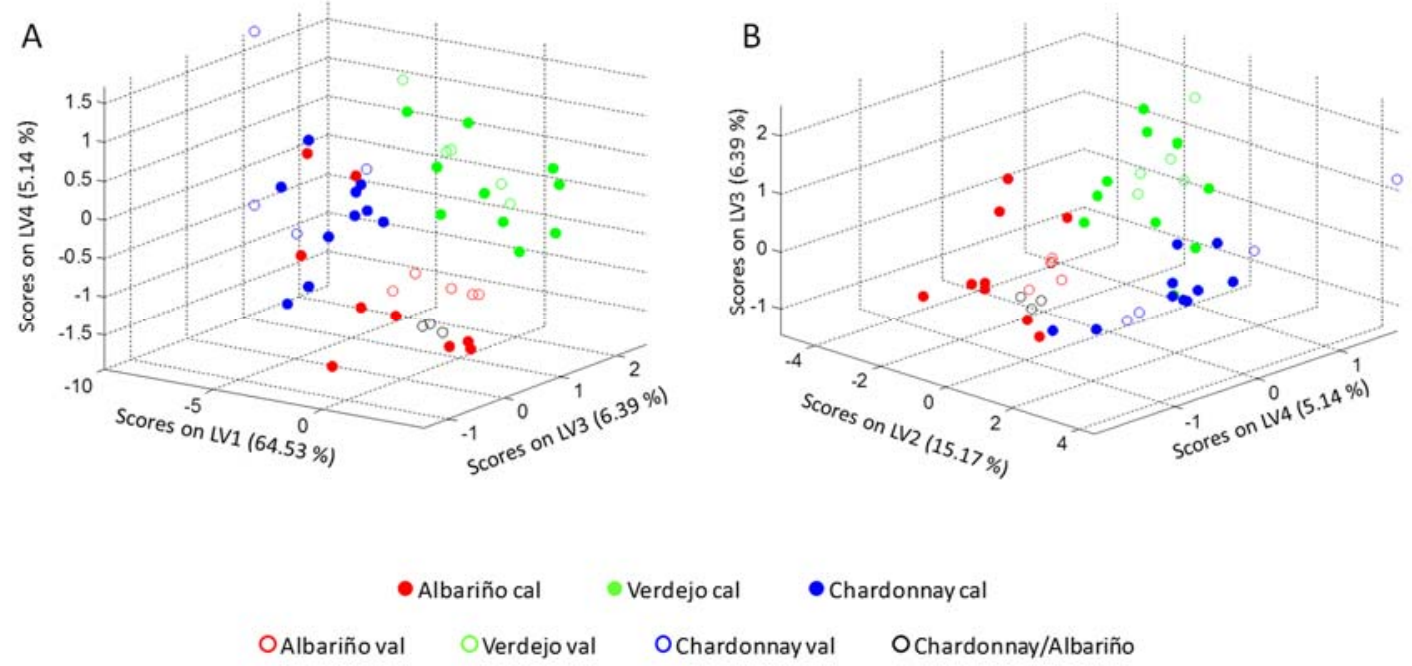

Figure 4. 3D scores plot obtained after PLS-DA models built from white wine HPLCEC signals. (A) LV1, LV3 and LV4 or (B) LV2, LV3 and LV4 are considered. Calibration (cal) and validation (val) sets are represented by filled and empty symbols respectively. 